\title{
Das Problem der beruflichen Bildung bei Adam Smith und den englischen Utilitaristen
}

\author{
Thomas Deißinger
}

Universität Konstanz

\section{$1 \quad$ Einleitung}

Die englische Berufsausbildung kann als ein dezentralisiertes, aufgrund des besonderen Gewichts der einzelbetrieblichen Gestaltung von Qualifizierungsprozessen äußerst heterogenes „System" beschrieben werden, das sich bislang trotz innovativ anmutender Reformkonzepte mit Erfolg der externen Reglementierung - insbesondere von staatlicher Seite - erwehrt hat. Das hier vorherrschende Grundmuster wurde jedoch im wesentlichen bereits von den sozio-ökonomischen Rahmenbedingungen der Industriellen Revolution geformt $^{1}$. In der Geisteshaltung einer im Calvinismus wie auch in der angelsächsischen Aufklärung wurzelnden Denktradition liegt eine der historischen „Ursachen" dieses englischen „Sonderweges". Indikatoren für eine „retardierte" Sozialstaatlichkeit im Vergleich mit Deutschland lassen sich hierbei vor allem in zwei Bereichen identifizieren: Zum einen was den öffentlichen Umgang mit der "sozialen Frage" des 19. Jahrhunderts betrifft; zum zweiten was die Entstehung eines Staatsverständnisses angeht, das insoweit vom deutschen Muster divergierte, als Umrisse eines „bürokratischen Staates“ nur zögerlich und vor dem Hintergrund einer bereits über ein halbes Jahrhundert hinweg verlaufenen industriellen Entwicklung entstanden ${ }^{2}$. Mit ihrem Bekenntnis zum „freien Markt" fundierte die Klassische Nationalökonomie jenes liberale Grundmuster, welches der praktizierende Puritanismus dem Verhaltenskodex des einzelnen Bürgers als übergreifende Verbindlichkeit gesellschaftlicher Verhaltensweisen bereits seit dem ausgehenden 16. Jahrhundert vorgeschrieben hatte. Für die liberalen Denker war unstrittig, daß es sich bei der Wirtschaft um eine „Domäne der Sittlichkeit“3 handelte und der Staat als Garant der individuellen Rechte, jedoch nicht als Interventionsstaat eine Existenzberechtigung hatte. Dennoch kann gleichzeitig von einer ambivalenten Haltung hinsichtlich der Legitimität sozialpolitischer und bildungspolitischer Aktivitäten der öffentlichen Hand gesprochen werden: Während die klassischen Vertreter der Nationalökonomie für den wirtschaftlichen Bereich die Zurückhaltung der Regierung reklamierten, betonten insbesondere die Utilitaristen die Bedeutung einer vom Staat zumindest mitgestalteten Erziehung des Volkes. Vergleichsweise unpräzise bleiben demgegenüber die

\footnotetext{
${ }^{1}$ Vgl. Deißinger (1992), (1994) und (1999).

${ }^{2}$ Vgl. Rohe (1984), S. 167 ff. passim.

${ }^{3}$ Freyer (1966), S. 113.
} 
Überlegungen zum Problem der beruflichen Bildung, was die Vermutung einer starken „Vergangenheitsabhängigkeit“ der aktuellen Strukturen auch in ideengeschichtlicher Hinsicht unterstreicht.

\section{Der bildungsökonomische Charakter der Gedanken über Bildung und Berufsbildung bei Adam Smith und John Stuart Mill}

Die Bedeutung, die Adam Smith im Wealth of Nations der menschlichen Arbeit und ihrer Produktiviät beimißt, zeigt sich darin, daß Smith sein Werk mit Überlegungen über die Arbeitsteilung beginnt. Dabei zeitigt diese nach Smiths Ansicht nicht nur quantitative Ergebnisse, die sich in einer Steigerung der Arbeitsproduktivität niederschlagen. Auch die qualitativen Aspekte werden in Smiths Aufzählung jener Faktoren genannt, die in seinen Augen zu einer effizienteren Arbeitsleistung führen: größere Geschicklichkeit, Innovations- und Erfindungsfreudigkeit, Zeitersparnis. Auf sie rekurriere die Tatsache, „daß es überhaupt zu verschiedenen Berufen $\mathrm{kam}^{\text {"4 }}$. Mit der Charakterisierung des natürlichen Tauschtriebes als Ursache der Arbeitsteilung verknüpft Smith die Vorstellung, der Einzelne werde im Rahmen des ,richtigen“ Marktverhaltens seinen Beruf pflegen und - im eigenen Interesse - nach Kräften fördern. Auf den ersten Blick scheint hier das egoistische Motiv vorzuherrschen. Aber es geht Smith auch um die kollektive Verantwortung, von der das gesamte ökonomische System abhängt, da ja alle Berufe zum Wohle des sozialen Ganzen beitragen. Smith gibt sich überzeugt, „daß olıne Mithilfe und Zusammenwirken Tausender von Menschen in einem zivilisierten Land nicht einmal der allereinfachste Mann ... versorgt werden kömnte ${ }^{\text {"5 }}$. Hierbei betont Smith, daß die auf dem Wege der Sozialisation des Einzelnen in der arbeitsteilig strukturierten Wirtschaft erworbenen „Talente“ und Fähigkeiten „meist mehr Folge als Ursache der Arbeitsteilung“ seien. Somit komme „Lebensweise, Gewohnheit und Erziehung" eine wesentliche Bedeutung zu${ }^{6}$. Das Prinzip des selbsttätig und eigenverantwortlich agierenden Individuums und ein vom Gemeinwohl her begründeter funktionalistischer Berufsbegriff sind somit im Wealth of Nations unmittelbar aufeinander bezogen.

Smith betont die Bedeutung und die Entwicklungsfähigkeit des Human Capital als Produktionsfaktor und dessen Funktion innerhalb des „Systems der natürlichen Freiheit "7. Wir haben es hier mit einer betont bildungsökonomischen Argumentation zu tun, was durch die folgende Aussage Smiths unterstrichen wird:

"Von einer teuren Maschine muß man erwarten, daß sie bis zu ihrem

Verschleiß einen außergewöhnlichen Ertrag abwirft, der ausreicht, das

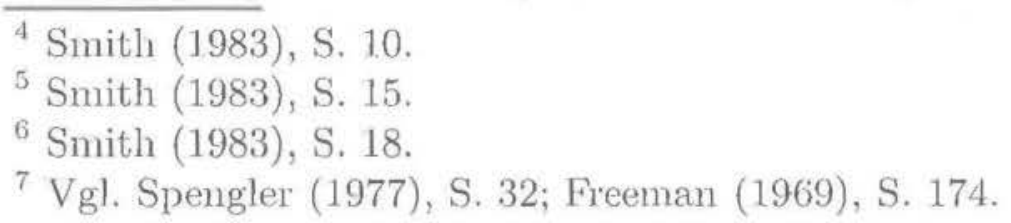


investierte Kapital zu ersetzen und zumindest einen normalen Gewinn zu zahlen. Mit einer solch aufwendigen Maschine kann man nun auch einen Menschen vergleichen, der mit großem Aufwand an Mühe und Zeit für eine Beschäftigung ausgebildet wurde, die außergewöhnliches Geschick und Fachkenntnis erfordert. Denn man sollte erwarten, daß er aus seinem erlernten Beruf einen Ertrag erzielen kann, der so weit über dem üblichen Lohn für einfache Arbeit liegt, daß er ihm den gesamten Ausbildungsaufwand, nebst einem normalen Gewinn für ein gleichwertiges Kapital, ersetzt. Auch muß er dieses Entgelt in angemessener Zeit verdienen, wobei man die äußerst ungewisse Lebenszeit ähnlich berücksichtigen muß wie die besser abschätzbare Nutzungsdauer der Maschine. Auf diesem Prinzip beruht der Lohnunterschied zwischen gelernter und ungelernter Arbeit." 8

Dem Beruf kommt bei Smith somit die Bedeutung eines ökonomischen Gutes zu, wobei das arbeitende Individuum wie der Kapitalinvestor einen naturgegebenen Anspruch darauf hat, für seine Ausbildungsausgaben entschädigt zu werden. Obgleich dieser bildungsökonomische Aspekt letztlich in Smiths Denken keine weitere explizite Ausgestaltung erfährt ${ }^{9}$, bedeutet dies mitnichten, daß er dem Thema einer qualifizierten beruflichen Unterweisung sowie einer gründlichen Erziehung keine Aufmerksamkeit gewidmet hätte. Dies zeigt seine Differenzierung der „gelernten" und „ungelernten" Arbeit. Vor diesem Hintergrund überrascht allerdings das Fehlen konstruktiver Ausführungen zur Frage beruflicher Qualifizierung. Dies dürfte vor allem mit Smiths Kritik gegenüber den merkantilistischen Hemmnissen seiner Zeit, die mit seiner Vorstellung einer freien wettbewerbsorientierten Wirtschaft unvereinbar waren, zu tun haben ${ }^{10}$.

Smith führt die hauptsächlichen Restriktionen, die die Ausgestaltung der durch Arbeitsteilung zustande kommenden berufsspezifischen Differenzierung sowie die Unterwerfung des Humankapitals unter die Marktgesetze behindern, auf die folgenden Ursachen zurück ${ }^{11}$ : die Macht der Zünfte und die Regelungen des Lehrlingsstatuts von $1563^{12}$; die Einmischung des Staates in die Berufsausbildung; die mobilitätshemmenden Armengesetze und die Niederlassungsgesetzgebung. Die „ausschließenden Privilegien der Zünfte“ “13 sind nach Smith verantwortlich dafür, daß der freie Markt im Handwerk nach wie vor Behinderungen unterworfen sei und auf diese Weise die freie Entfaltung individueller Anstrengungen unterdrückt werde. Smith wendet sich gegen die Länge der handwerklichen Lehre, die durch das Lehrlingsstatut zwingend vorgeschrieben war, und plädiert statt dessen für die Aufhebung der Begren-

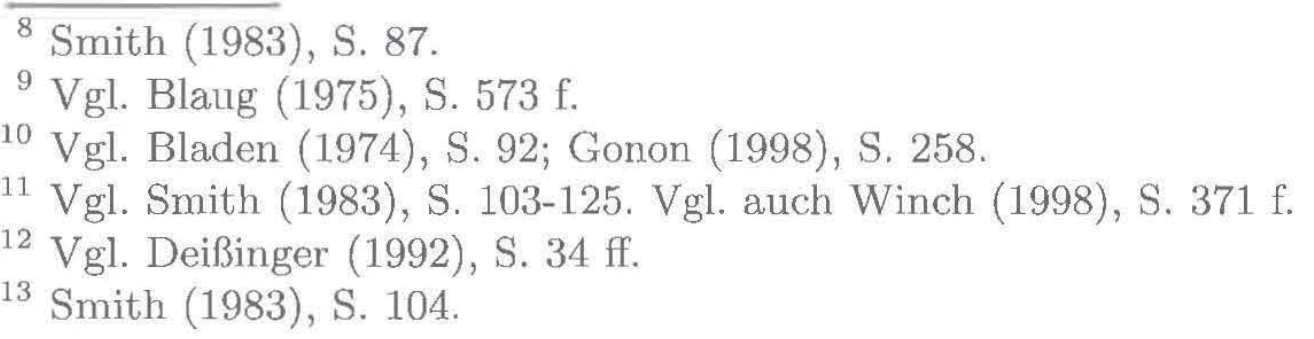


zung der Lehrlingszahl in den einzelnen Gewerben sowie für die frülızeitige Möglichkeit der in ein Gewerbe eintretenden jungen Mämmer, als Gesellen arbeiten zu dürfen. Thre Arbeit sei um der Geschicklichkeit und der handwerklichen Kompetenz willen auf das Leistungsprinzip zu verpflichten. Neben den den freien Wettbewerb behindernden Restriktionen kritisiert Smith auch die Ausbildung für die geistigen und geistlichen Berufe: Sie impliziert in seinen Augen ein marktverzerrendes Moment, weil das auf diesem Wege künstlich erzeugte Überangebot an Arbeitskräften einen Arbeitsmarktausgleich und damit die volkswirtschaftliche Harmonie der individuellen Marktinteressen verhindere $^{14}$. Schließlich fordert Smith die Eliminierung sozialer und beruflicher Mobilitätsschranken: Die Armen- und Niederlassungsgesetze verfolgten nur den Zweck, Erwerbslosen in Regionen mit vorhandener Nachfrage nach Arbeit Aufnahme und Beschäftigung zu verwehren. Unübersehbar verbinden sich hier Smiths Forderungen nach Abbau der überkommenen Ausbildungs-, Lohn- und Mobilitätsrestriktionen mit der Vorstellung einer möglichst effizienten Ausrichtung der beruflichen Ausbildung ${ }^{15}$. Smiths Ablehnung staatlicher Steuerung der Berufserziehung beruht deshalb primär auf seiner Abneigung gegenüber jeglicher Reglementierung des Erwerbs- und Wirtschaftslebens.

Berufliche Bildung konstituiert zwar nach Smith in qualitativer Hinsicht den materiellen Progreß der Volkswirtschaft. Zu einem nicht weniger wichtigen Thema der Smithschen Ausführungen zum Zusammenhang von Wirtschaft und Bildung wird allerdings der Gedanke der Elementarbildung ${ }^{16}$. Sieferle merkt hierzu an, daß Smith selbst in seinem arbeitsteiligen Konzept „einen groben Schönheitsfehler entdeckt" habe ${ }^{17}$ : die Deprivation des Arbeiters. Ihr möchte er mit pädagogischen Mitteln begegnen. Smith gibt deshalb auch der "Erziehung des einfachen Volkes" Vorrang gegenüber der Oberschicht. Er verweist auf die existentielle Bedeutung beruflicher Arbeit und damit auf die Unmöglichkeit pädagogisch verantworteter Erziehung und Ausbildung bei den ,jungen Leuten aus der unteren Schicht“. Hier könne die Regierung helfen, inden ,sie in jeder Gemeinde oder jedem Distrikt eine bescheidene Schule errichtet, in der die Kinder gegen ein geringes Schulgeld unterrichtet werden, das auch der einfache Arbeiter aufbringen kann"18. Smith empfiehlt diesbezüglich eine über Lesen, Schreiben und Rechnen hinausgehende Einbeziehung praktischer Fächer wie Geometrie und Mechanik. Auch reklamiert Smith für die Elementarerziehung eine staatliche Schulabschlußprüfung, von der die Aufnahme in ein Gewerbe abhängig gemacht werden solle. Dabei sei es unerheblich, ob sich an die Phase der schulischen Bildung eine Berufsausbildung in einer Zunft anschließe oder ob die Prüfung direkt

\footnotetext{
${ }^{14}$ Vgl. Smith (1983), S. 106-117.

${ }^{15}$ Vgl. Spengler (1977), S. 35 f.

16 Vgl. Freeman (1969), S. 174.

${ }^{17}$ Vgl. Sieferle (1984), S. 119.

${ }^{18}$ Smith (1983), S. 664 f.
} 
dazu führe, daß der Einzelne „in einem Dorf oder in einer Stadtgemeinde ein Gewerbe betreiben darf"19. Hierbei verweist Smith auch auf den Nutzen, der dem Staat aus der Erziehung des Volkes erwachse ${ }^{20}$. Vor diesem Hintergrund schließe ich mich der Charakterisierung Hollander's an, der auf Smith bezogen folgende Gesichtspunkte seines Erziehungsdenkens hervor hebt ${ }^{21}$ :

- Der Optimismus Smiths in die Mobilitätsfähigkeit des arbeitenden Menschen in einer freien Wirtschaft gründet auf der Einsicht, hierzu könne auch die Erziehung und Bildung des Einzelnen beitragen, wobei auch dem Staat eine Verantwortung zugewiesen wird.

- Die Vorbehalte Smiths gegen den Staat richten sich vor allem gegen die merkantilistische Wirtschaftspolitik und die korporativen Vorrechte der Zünfte und Monopole des 17. und 18. Jahrhunderts, die er im Interesse eines freien Marktes eliminieren möchte.

- Auch wenn sich Smith nicht für öffentliche berufliche Bildungseinrichtungen einsetzt, darf dies nicht als Feindseligkeit gegenüber beruflicher Unterweisung und Ausbildung schlechthin gewertet werden.

- Letzten Endes bleiben Smiths Ausführungen jedoch unpräzise und unschlüssig hinsichtlich der Frage, auf welche Weise und in welchem Rahmen eine technisch-berufliche Ausbildung durchzuführen sei.

Smiths Idee der Volksbildung steht keineswegs im Widerspruch zu seinen Auffassungen einer liberalisierten Wirtschaftsordnung ${ }^{22}$. Tatsächlich erkennt Smith in der Bereitstellung von Schulen eine wichtige Rahmenbedingung für das Wohlergehen einer Gesellschaft. Die ökonomische Dimension der staatlichen Erziehung bestehe darin, daß diese ihren Beitrag zum Wohle des Einzelnen wie der Gemeinschaft zu erbringen habe ${ }^{23}$. Smiths zweckrationaler Bildungsvorstellung entspricht die Forderung, Bildungsinstitutionen einem ökonomischen Konkurrenzprinzip zu unterwerfen sowie Qualität und Effizienz des Bildungsangebots in den öffentlichen Erziehungseinrichtungen zu stärken. Angesichts der kritischen Auseinandersetzung mit der herrschenden institutionalisierten Ordnung seiner Zeit erscheinen Smiths Ideen zur Ausgestaltung eines effizienten Elementarerziehungswesens weitsichtig. Andererseits wollte er jedoch die Unterwerfung der praktischen Berufsausbildung unter die Gesetze des Marktes. Sieferle weist darauf hin, daß es sich bei der Befürwortung des Eingreifens einer „außerökonomischen Instanz“, der Schaffung öffentlicher Bildungseinrichtungen, um eine „beachtliche Konzession" des Ökonomischen Liberalismus gehandelt habe ${ }^{24}$. Jedoch habe Smith einen staatlichen Beitrag nicht aus "Menschenfreundlichkeit" empfohlen, sondern weil es ihm um die

${ }^{19}$ Smith (1983), S. 665.

${ }^{20}$ Vgl. Smith (1983), S. 667 f.

${ }^{21}$ Vgl. Hollander (1967/68), S. 524 f.

${ }^{22}$ Vgl. Gonon (1998), S. 254.

${ }^{23}$ Vgl. Osterwalder (1993), S. 57.

${ }^{24}$ Vgl. Sieferle (1984), S. 119. 
Ermöglichung der Eigeninitiative des wirtschaftenden Menschen ging ${ }^{25}$. Die Feststellung, es könne „keine Nation blühen und gedeihen, deren Bevölkerung weithin in Armut und Elend lebt ${ }^{* 26}$, basiert einerseits auf einem differenten Menschenbild im Vergleich mit den Sozialtheoretikern der Aufklärung und des Frühliberalismus ${ }^{27}$. Andererseits darf jedoch Smiths Befürwortung einer. vom Staat zumindest mitgetragenen Erziehung des Volkes nicht als prinzipieller Aufbruch zur Sozialstaatlichkeit gewertet werden. Ein solcher verbot sich nicht zuletzt aus dem Glauben an das Individuum und die Macht der „unsichtbaren Hand" ${ }^{28}$, aber auch aus der grundsätzlichen Abneigung der. Klassischen Ökonomie gegen das „merkantilistische System“.

Auch John Stuart Mills Kritik am Erziehungssystem Englands um die Mitte des 19. Jahrhunderts hielt diesen nicht davon ab, vor einer zu dominanten Rolle des Staates zu warnen: Der Staat sei nur dann ein angemessener Sachwalter des Gemeinwohls, wenn sich seine Institutionen als unauthoritative erwiesen und im Hinblick auf die mental cultivation des einzelnen Bürgers lediglich eine unterstützende Aufgabe wahrnähmen ${ }^{29}$. Bei Mill steht unverkennbar der bildungsökonomische Aspekt im Vordergrund. Die "technische“ und „industrielle" Erziehung - so Mill - werde vor allem deshalb unternommen, um dadurch größeren und wertvolleren Ertrag zu erzielen:

"To the community at large, the labour and expense of rearing its infant population form a part of the outlay which is a condition of production, and which is to be replaced with increase from the future produce of their labour. By the individuals, this labour and expense are usually incurred from other motives than to obtain such ultimate return, and, for most purposes of political economy, need not be taken into account as expenses of production. But the technical or inclustrial education of the community; the labour employed in learning and in teaching the arts of production, in acquiring and communicating skill in those arts; this labour is really, and in general solely, undergone for the sake of the greater or more valuable produce thereby attained, and in order that a remuneration, equivalent or more than equivalent, may be reaped by the learner, besides an adequate remuneration for the labour of the teacher, when a teacher has been employed." 30

Zu den Hauptthemen der Politischen Ökonomen zählten somit neben der Erziehung in ilurer Funktion als Kapitalfaktor vor allem das Problem der Effizienz von Bildungsinstitutionen und damit die Frage nach der Rolle des Staates

${ }^{25} \mathrm{Vgl}$. Winch $(1998)$, S. 366.

26 Smith (1983), S. 68.

27 So formulierte Mandeville (1755), S. 256 in seiner Bienenfabel: ,... in a free nation where slaves are not allowed of, the surest wealth consists in a multitude of laborious poor".

${ }^{28}$ Vgl. Baumol (1991), S. 27 f.

${ }^{29}$ Vgl. Garforth (1980), S. 118 f.

${ }^{30}$ Mill (1968), S. 41. 
hinsichtlich der Bereitstellung und Verbesserung von Bildungsmöglichkeiten. Während bei Smith und John Stuart Mill Erziehung und Ausbildung unter dem Aspekt der subjektiven Nützlichkeit und Verwertbarkeit behandelt werden, stützen Jeremy Bentham und James Mill, die Vertreter des frühen Utilitarismus, ihren Erziehungsoptimismus auf die positive Funktion des Erziehungswesens im Hinblick auf die Beförderung des „größten Glücks der größten Zahl".

\section{Erziehung als Voraussetzung für das „größte Glück der größten Zahl": Zur Erziehungstheorie bei Jeremy Bentham und James Mill}

Jeremy Bentham's Erziehungslehre kreist um den Begriff der Chrestomathia (useful learning) - Titel einer 1816 veröffentlichten Erziehungsschrift ${ }^{31}$. Es ist der Versuch, die Ausformung eines anti-traditionalistischen Erziehungsprogramms mit einer Modellschulkonzeption zu verbinden. Vor allem unter didaktischem Aspekt erweisen sich Bentham's Vorstellungen als fortschrittlich, obgleich sich seine Chrestomatic School in schulorganisatorischer und methodischer Hinsicht am Bell-Lancasterschen Schultypus orientiert ${ }^{32}$, den die Utilitaristen aufgrund seiner Rationalität und ökonomischen Effizienz guthie$ß_{\mathrm{en}}^{33}$. Das allgemeine Erziehungsziel Bentham's knüpft unmittelbar an seiner Moralphilosophie an: „Education is a series of conduct directed to an end; ... The common end of every person's education is Happiness“"34. Die Aufgabe der Regierung - so Bentham - bestehe darin, effiziente Bildungseinrichtungen zu gründen, die dieses Glück zu befördern hätten. Bentham glaubte nicht an eine prästabilierte Harmonie des gesellschaftlichen Zustands, sondern setzte voraus, daß dieser gesteuert werden müsse: „... by rational planning and by science 335 . Dieser Idee sind vor allem die praktischen Reformer unter den Utilitaristen gefolgt, die es als politische Aufgabe ansahen, „schlechte“ Institutionen durch "gute", nicht funktionierende durch effiziente zu ersetzen. Dabei sah Bentham in der "Regierungskunst" und in der "Erziehungskunst" etwas Gleichwertiges: Da das öffentliche Wohl Gegenstand der Gesetzgebung sei, müsse auch die Erziehung öffentliche Angelegenheit sein. Beide seien auf das „Prinzip der Nützlichkeit“ zu verpflichten: „The magistrate may be considered as a national instructor" ${ }^{66}$. Eine „Aufklärung“ und „Kultivierung“ des Volkes sei mit Blick auf das Wahlrecht, die Rückführung von Delinquenten in die Sozialgemeinschaft und angesichts der Notwendigkeit, die Kinder

\footnotetext{
31 Vgl. Bentham (1962).

${ }^{32} \mathrm{Vgl}$. Hans (1952), S. 90 ff.

33 Vgl. Rössner (1984), S. 65 f.

34 Zitiert bei Hans (1952), S. 93.

35 West (1975), S. 127.

36 Bentham (1975), S. 25.
} 
der Armen zu nützlichen Staatsbürgern zu erziehen, unerläßlich. Eine fundierte, allen Bürgern zugute kommende intellektuelle, soziale und berufliche Bildung und Ausbildung sei nicht zuletzt unabdingbar für die Wirksamkeit „guter Gesetze“37.

Bentham's didaktisch-pädagogisches Denken beruht maßgeblich auf einem naturwissenschaftlich-pragmatischen Bildungsverständnis. Seine Ausarbeitung eines didaktischen Plans (encyclopaedic table) ist hierbei von zwei Ordnungskriterien bestimmt: Es handelt sich um die Variablen "Utilität" und „Komplexität". Die erste steht für die Anwendbarkeit der Bildungsinhalte auf das praktische Leben; die zweite impliziert, daß die Bildung des Menschen induktiv - wie Bacon dies vorschwebte - erfolgen, d.h. vom Einfachen zum Komplexen, vom Besonderen zum Allgemeinen fortschreiten sollte. Bemerkenswert ist hierbei auch die Eimbeziehung eines vocational principle. Allerdings versteht Bentham dieses nicht als Einengung auf eine berufliche Spezifizierung, sondern er weist ihm die Funktion zu, im Rahmen des Lemprozesses ein technisch-wissenschaftliches Verständnis zu erzeugen, das einer späteren beruflichen Tätigkeit zugute kommen solle. Nicht vergessen werden darf jedoch, daß Bentham das Erziehungssystem auch als Ordnungsfaktor für das Gemeinwesen ansahr ${ }^{38}$.

Bentham's Leistumg liegt in der Grundlegung einer utilitaristischen Erziehungslehre. Es war James Mill, der „systematisierte und zusammenfaßte, was auf dem Stande der Chrestomathia utilitaristische Pädagogik war “39. Der Ausgangspunkt ist der gleiche wie bei Bentham: ,The end of education is to render the individual, as much as possible, an instrument of happiness, first to himself, and next to other beings ${ }^{40}$. Mill's erzieherisches Denken ist vor diesem Hintergrund in zweifacher Hinsicht bedeutsam:

- Zurn ersten verfolgte Mill das Ziel einer wissenschaftlichen Behandlung der Erziehung als eines gesellschaftlichen Problems, im Sinne einer sowohl ethischen als auch pädagogisch-psychologischen Fundierung ${ }^{41}$.

- Zum zweiten war Mill's Erziehungstheorie direktes Resultat seiner politischen und sozialphilosophischen Lehre, in der das Aufbrechen der Interessenallianz von Aristokratie, Kirche und middle class in Erziehungsfragen $^{42}$ und die „demokratische Neuorientierung“ der Erziehung eingefordert wurden.

In seinem Essay on Education aus dem Jahre 1818 orientiert sich Mill hinsichtlich der Perfektionierbarkeit der menschlichen Natur an Locke und Smith, konkretisiert jedoch die Idee einer „nützlichen Erziehung“, indem er eine „demokratische" Regierung und ein öffentliches Erziehungswesen als wesentliche

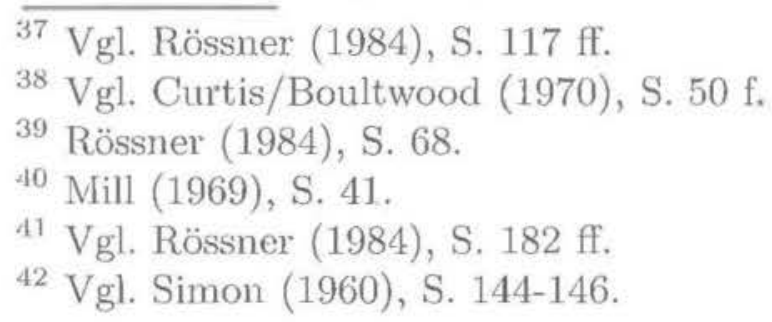


Voraussetzungen für die „Glückseligkeit“ eines Gemeinwesens anführt³. Damit das Individuum zu dieser Glückseligkeit befähigt wird, hat die Erziehung kognitive und moralische "Qualitäten“ zu vermitteln (qualities of mind): Intelligenz (intelligence), Mäßigung (temperance), Großzügigkeit (generosity) und Gerechtigkeit (justice). Diese korrespondieren mit den vier Bereichen der Erziehung: domestic education, technical education, social education, political education ${ }^{44}$. Ein direktes Zuordnungsverhältnis besteht nach Mill zwischen dem Bereich der technical education, der sowohl die allgemeine schulische als auch die berufliche Erziehung umfaßt, und der kognitiven Dimension der „Intelligenz", deren Elemente „Wissen“ (knowledge) und „Klugheit" (sagacity) $\operatorname{sind}^{45}$. Der Begriff bezieht sich vor dem Hintergrund der hedonistischen Zielsetzung des Utilitarismus auf die intellektuelle Leistungsfähigkeit des Menschen, die als individuelle Voraussetzung für ein vorbildliches Gemeinwesen und das "größte Glück der größten Zahl“ angesehen wird. Jeder Akt der Erziehung wirkt nach Mill in zweifacher Hinsicht: „When the consequences of the act are pleasurable to other persons ... the intention ... is good ${ }^{46}$. Der individuelle Nutzen der Erziehung verbindet sich mit den sozialen Vorteilen zu einem Mittel-Zweck-Verhältnis.

In diesem Sinne gehen Bildungsoptimismus und Bildungspragmatismus im Denken Mill's ineinander über, gleichzeitig geht es um den Gleichheitsund Demokratiegedanken, mit dem das utilitaristische Bildungsverständnis eine politische Dimension erhält: „As we strive for an equal degree of justice, an equal degree of temperance, an equal degree of veracity, in the poor as in the rich, so ought we to strive for an equal degree of intelligence, ... "47. Mill geht es um formale „Chancengleichheit", ein Anliegen, das vor ihm Smith und Bentham und nach ihm sein Sohn John Stuart Mill in gleicher Weise vertreten ${ }^{48}$. Er fordert deshalb eine „allgemeine Volksbildung, Intelligenzförderung für alle, schools for all ${ }^{49}$, wobei jegliche Erziehung lebenspraktisch, berufsbezogen und nützlich sein sollte. Dieses „Individualitätsprinzip" der Erziehung beruht auf der Einsicht in die Notwendigkeit staatlich verantworteter Erziehung für das ganze Volk. Denn da Erziehung als „Kunst der Glückseligkeit" definiert wird, muß sie jedes Individuum einbeziehen. Die faktische pädagogische Monopolstellung des Anglikanismus und die Gegnerschaft der Kirche zu den säkular und anti-dogmatisch orientierten Schulen des Dissent, denen Bentham und Mill nahestanden, seien - so Mill - für die Defizite des englischen Erziehungswesens im Vergleich zum funktionierenden öffentlichen „System“ Irlands und Schottlands verantwortlich ${ }^{50}$.

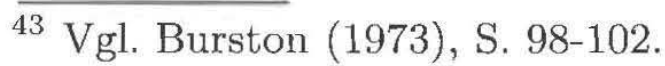

${ }^{44}$ Vgl. Rössner (1984), S. $181 \mathrm{f}$.

${ }^{45}$ Vgl. Hans (1952), S. 97-99.

46 Zitiert bei Burston (1973), S. 106.

47 Mill (1969), S. 106.

${ }^{48}$ Vgl. Miller (1966), S. 307.

49 Rössner (1984), S. 199.

${ }^{50}$ Vgl. Mill (1813), S. $211 \mathrm{ff}$.
} 
Indem sie auch dem Arbeiter, den Unterschichten und somit dem ganzem Volk die Bildungswïrdigkeit zusprachen, brachen die Utilitaristen mit der klerikal-aristokratischen, aber auch mit der puritanischen und aufgeklärtfrühliberalen Tradition. Eine ,realistische“, auf das Säkulare verpflichtete, in die gesellschaftlichen Belange integrierte ,nützliche“ Erziehung sahen sie als unabdingbare Voraussetzung für den gesellschaftlichen Fortschritt.

Bemerkenswert ist, daß die erziehungsgeschichtliche Entwicklung des viktorianischen England nur zögerlich den Spuren der Utilitaristen sowie jener sozialen und politischen Kräfte folgte, die die Idee der Erziehung des Volkes im Kontext „sozialstaatlicher Verantwortlichkeit“ zu verankern suchten ${ }^{51}$. Dies kann nur damit erklärt werden, daß die gesellschaftsbeeinflussende Kraft des klassischen Wirtschaftsliberalismus weit stärker war als die politisch fortschrittlichen Gedanken der utilitaristischen Philosophie. Konstruktive Gedanken zur Lehrlingserziehung im besonderen bzw. zur Berufserziehung im allgemeinen finden wir in beiden Konzepten nur am Rande.

\section{Schlußbemerkungen}

Erziehung und Ausbildung können nicht losgelöst von der Geschichte eines Landes, seiner gesellschaftlichen Entwicklung sowie seinen Institutionen betrachtet werden. Die Hauptunterschiede zwischen der deutschen und der englischen Entwicklung liegen in der gesellschaftlichen Behandlung der Lehrlingsfrage in der Phase der Hochindustrialisierung. In Deutschland wurde das Handwerk just in jener Zeit vor allem deshalb zum normierenden Faktor der gewerblichen Ausbildung ${ }^{52}$, weil sich das liberalistische Denken in Deutschland nicht den Raum wie in anderen Ländern verschaffen konnte und das Gewicht des konservativen Legitimationsmusters beruflicher Erziehung sowohl im Zeichen wachsender Gewerbefreiheit als auch später im 20. Jahrhundert die Wiederaufnahme anti-industrieller Zielpositionen ermöglichte ${ }^{53}$. Dies garantierte das Überleben der "Meisterlehre", die in England heute nur noch eine randständige Rolle im beruflichen Bildungssystem spielt. Der Berufspädagoge Abraham kennzeichnet überdies die „Unterscheidung zwischen der betrieblichen und der schulischen Berufserziehung in Deutschland" als eine Lösung des Berufsbildungsproblems, die auf Denkvoraussetzungen beruhte, die in anderen Ländern nicht oder jedenfalls nicht in der gleichen Weise existierten $^{54}$.

Ich möchte mit einem Zitat von Christopher Winch abschließen:

"Therefore the changing of a VET system, just like the changing of an education system more generally, is dependent upon and also has

51 Vgl. Deißinger (1992), S. $201 \mathrm{ff}$.

52 Vgl. Blankertz (1969), S. 127.

${ }^{53}$ Vgl. Stratmann (1982), S. 183 ff.; Gonon (1998), S. 260 f.

${ }^{54}$ Vgl. Abraham (1962), S. $171 \mathrm{f}$. 
ramifications throughout society and politics. It cannot be a simple matter of a technical recipe to aid economic growth, but touches on the heart of what any society is about." 55

Aus der "Entscheidung" Englands für den Liberalismus ergab sich der Rahmen, der das einer Legitimation zuführbare Funktionsspektrum „sozialstaatlicher Verantwortlichkeit" im 19. Jahrhundert absteckte. Es ist deshalb angemessen, den Wirtschaftsliberalismus und seine theoretische Behandlung des Produktionsfaktors "Arbeit" als kulturgeschichtlich signifikante Größe der englischen Entwicklung herauszustellen ${ }^{56}$. Seine Repräsentanten haben mit ihrer insgesamt ambivalenten Haltung zur Rolle des Staates ihre Spuren auch im angelsächsischen System der beruflichen Bildung hinterlassen.

\section{Literaturverzeichnis}

Abraham, K. (1962) Die Aufgaben der Vergleichenden Internationalen Wirtschaftspädagogik. Zeitschrift für Pädagogik 8, 166-178.

Baumol, W.J. (1991) A Glimpse of the Invisible Hand. In: Shaw, G.K. (Ed.): Economics, Culture and Education. Essays in Honour of Mark Blaug. Aldershot, 27-36.

Bentham, J. (1962) Chrestomathia (1816). In: Bowring, J. (Ed.): The Works of Jeremy Bentham, Vol. Eight, reprinted. New York, 1-191.

Bentham, J. (1975) The Theory of Legislation (1802). With an introduction by U. Baxi. Bombay.

Biernacki, R. (1995) The Fabrication of Labor. Germany and Britain, 16401914. Berkeley.

Bladen, V. (1974) From Adam Smith to Maynard Keynes: The Heritage of Political Economy. Toronto.

Blankertz, H. (1969) Bildung im Zeitalter der großen Industrie. Pädagogik, Schule und Berufsausbildung im 19. Jahrhundert. Hannover.

Blaug, M. (1975) The Economics of Education in English Classical Political Economy: A Re-Examination. In: Skinner, A., Wilson, Th. (Eds.): Essays on Adam Smith. Oxford, 568-599.

Blaug, M. (1985) Adam Smith und die Organisation der Bildung. In: Recktenwald, H.C. (Hrsg.): Ethik, Wirtschaft und Staat. Darmstadt, 257-261. Burston, W.H. (1973) James Mill on Philosophy and Education. London. Curtis, St.J., Boultwood, M.E.A. (1970) An Introductory History of English Education since 1800, 4th ed. (reprinted). London.

Deißinger, Th. (1992) Die englische Berufserziehung im Zeitalter der Industriellen Revolution. Ein Beitrag zur Vergleichenden Erziehungswissenschaft. Würzburg.

55 Winch (1998), S. 377.

56 Vgl. hierzu auch: Biernacki (1995). 
Deißinger, Th. (1994) The Evolution of the Modern Vocational Training Systems in England and Germany: A Comparative View. Compare 24, $17-36$.

Deißinger, Th. (1999) Beruflichkeit als Zusammenhang. Ein Vergleich mit England. In: Harney, K., H.-E. Tenorth (Hrsg.): Beruf und Berufsbildung. Situation, Reformperspektiven, Gestaltungsmöglichkeiten (40. Beiheft zur Zeitschrift für Pädagogik). Weinheim, 189-207.

Freeman, R.D. (1969) Adam Smith, Education and Laissez-faire. History of Political Economy 1, 173-186.

Freyer, H. (1966) Die Bewertung der Wirtschaft im philosophischen Denken des 19. Jahrhunderts (1921). Hildesheim.

Garforth, F.W. (1980) Educative Democracy: John Stuart Mill on Education in Society. Oxford.

Gonon, Ph. (1998) Berufliche Bildung und Gesellschaft in ökonomischer und pädagogischer Theoriebildung. In: Oelkers, J., F. Osterwalder, H. Rhyn (Hrsg.): Bildung, Öffentlichkeit und Demokratie (38. Beiheft zur Zeitschrift für Pädagogik). Weinheim, 251-266.

Hans, N. (1952) Bentham and the Utilitarians. In: Judges, A.V. (Ed.): Pioneers of English Education. A Course of Lectures Given at King's College London. London, 83-103

Hollander, S. (1967/68) The Role of the State in Vocational Training: The Classical Economists' View. Southern Economic Journal 34, 513-525.

Mandeville, B. de (1755) The Fable of the Bees: or; Private Vices, Public Benefits. With, An Essay on Charity and Charity Schools; and, A Search into the Nature of Society (1714/29), 9th ed. Edinburgh.

Mill, J. (1813) Education of the Poor. Edinburgh Review 2, 207-219.

Mill, J. (1969) Essay on Education (1818). In: Burston, W.H. (Ed.): James Mill on Education. London, 41-119.

Mill, J.St. (1968) Principles of Political Economy with Some of their Applications to Social Philosophy (1848), ed. by J.M. Robson. London.

Miller, W.L. (1966) The Economics of Education in English Classical Economics. Southern Economic Journal 32, 294-309.

Osterwalder, F. (1993) Markt, Staat, Öffentlichkeit und Bildung. In: Gonon, Ph.,J. Oelkers (Hrsg.): Die Zukunft der öffentlichen Bildung. Frankfurt a.M., $55-76$.

Rohe, K. (1984) Großbritannien: Krise einer Zivilkultur? In: Reichel, P. (Hrsg.): Politische Kultur in Westeuropa. Bürger und Staaten in der Europäischen Gemeinschaft. Frankfurt a.M., 167-193.

Rössner, L. (1984) Die Pädagogik der empiristisch-utilitaristischen Philosophie Englands im 19. Jahrhundert. Frankfurt a.M.

Sieferle, R.P. (1984) Fortschrittsfeinde? Opposition gegen Technik und Industrie von der Romantik bis zur Gegenwart. München.

Simon, B. (1960) Studies in the History of Education, 1780-1870. London. Smith, A. (1983) Der Wohlstand der Nationen. Eine Untersuchung seiner Natur und seiner Ursachen (1776), aus d. Englischen übertragen u. mit 
einer umfassenden Würdigung des Gesamtwerkes v. H.C. Recktenwald, 3. Aufl., München.

Spengler, J.J. (1977) Adam Smith on Human Capital. American Economic Review 67, 32-36.

Stratmann, K. (1982) Geschichte der beruflichen Bildung. Ihre Theorie und Legitimation seit Beginn der Industrialisierung. In: Blankertz, H. et al. (Hrsg.): Enzyklopädie Erziehungswissenschaft, Bd. 9.1: Sekundarstufe IIJugendbildung zwischen Schule und Beruf. Stuttgart, 173-202.

West, E.G. (1975) Education and the Industrial Revolution. London.

Winch, C. (1998) Two Rival Conceptions of Vocational Education: Adam Smith and Friedrich List. Oxford Review of Education 24 (3), 365-378. 